Cite this: Soft Matter, 2014, 10, 2759

Received 27th December 2013 Accepted 14th January 2014

DOI: $10.1039 / c 3 s m 53201 a$

www.rsc.org/softmatter

\title{
Frictional properties of a polycationic brush $\uparrow$
}

\section{Introduction}

Friction is a dissipative process that occurs whenever there is sliding contact between two surfaces. Early descriptions of friction by da Vinci and later formalized by Amontons ${ }^{1,2}$ have been remarkably robust. Although not explicitly stated in his manuscript, the work of Amontons in particular allows for a clear statement of the linear relationship between the frictional force, $F$ and load, $N$ (Amontons' law),

$$
F=\mu N,
$$

where $\mu$ is the coefficient of friction.

More formal approaches to friction have been attempted with some success. For example, a now classic paper on the adhesion of soft (elastic) solids formalized adhesion in a way that related to equilibrium thermodynamics. ${ }^{3}$ Similarly, further work allowed the adhesion between somewhat stiffer materials to be measured, ${ }^{4}$ which created a problem as to how to classify the different interactions. These two models, denoted by the authors' initials as JKR and DMT respectively are, in contrast to Amontons' law, single asperity models, which is necessary for a

${ }^{a}$ Department of Physics and Astronomy, University of Sheffield, Hounsfield Road, Sheffield S3 7RH, UK. E-mail: mark.geoghegan@sheffield.ac.uk; Fax: +44 (o)114 222 3555; Tel: +44 (0)114 2223544

${ }^{b}$ Department of Chemistry, University of Sheffield, Brook Hill, Sheffield S3 7HF, UK $\dagger$ Electronic supplementary information (ESI) available: Supporting information contains the adhesion (approach and retraction) data and friction-load plots for the brush films as a function of $\mathrm{pH}$ for all four functionalized tips. See DOI: 10.1039/c3sm53201a

\$ Present address: Department of Physics, George Washington University, Washington D.C. 20052, USA.

$\S$ Present address: Department of Chemical and Process Engineering, University of Strathclyde, Royal College Building, 204 George Street, Glasgow G1 1XW, UK.

I Present address: Hull Research and Technology Centre, BP Chemicals, Saltend Chemicals Park, Hull HU12 8DS, UK. thermodynamic foundation. The JKR and DMT approaches are explicitly dependent on the area of contact, whereas in Amontons' law, an area dependence is implicit due to increases in load yielding an increased contact area due to the effects of microscopic roughness. ${ }^{2}$ The JKR approach is appropriate for elastic and readily deformable surfaces (soft solids), whereas the DMT model is suitable for the analysis of the contact mechanics of stiff surfaces. Given the three different means to describe interactions, it is not always clear which to use. Relating JKR and DMT behaviour was a relatively easy problem to solve, with Carpick and colleagues developing the work of Maugis, ${ }^{5}$ and eventually describing the JKR and DMT approaches as two limiting cases through the general transition equation. ${ }^{6}$

For the JKR model, the area of contact is given by

$$
A=\pi\left(\frac{R}{K}\right)^{2 / 3}\left(N+3 \pi \gamma R+\sqrt{6 \pi \gamma R N+(3 \pi \gamma R)^{2}}\right)^{2 / 3},
$$

where $R$ is the radius of the spherical contact, $\gamma$ is the interfacial tension and $K$ is the (effective) elastic modulus of the system. Both surfaces can contribute to $K$, if both are deformable. The DMT model, however, gives the contact area as

$$
A=\pi\left(\frac{R}{K}\right)^{2 / 3}(N+4 \pi \gamma R)^{2 / 3} .
$$

Both these models reduce to that of Hertz ${ }^{7}$ when $\gamma=0$, i.e. there is no adhesive interaction between the two surfaces.

The general transition equation linking the JKR and DMT behaviours is given by ${ }^{6}$

$$
\left(\frac{a}{a_{0}}\right)=\left(\frac{\alpha+\sqrt{1-N / N_{\mathrm{PO}}}}{1+\alpha}\right)^{2 / 3},
$$

where $a$ is the contact radius when the load $N$ is applied, $a_{0}$ is the contact radius without load, $N_{\mathrm{PO}}$ is the force required to 
separate the two components (pull-off force), and $\alpha$ is the transition parameter, for which JKR behaviour is indicated by $\alpha=1$, and DMT behaviour by $\alpha=0$.

Polymers end-grafted to a substrate (known as brushes) have an important role in friction, adhesion, and lubrication, ${ }^{8-11}$ particularly when they involve charge. ${ }^{\mathbf{1 2 - 1 4}}$ In particular, polyelectrolyte brushes have the capacity to change their shape and properties in response to environmental $\mathrm{pH}$ or salt. ${ }^{15}$ For example, zwitterionic brushes of poly[2-(methacryloyloxy)ethyl phosphorylcholine] (PMPC) have been shown to be particularly lubricious, even when subjected to high pressures. ${ }^{16}$ PMPC brushes may nevertheless have their frictional behaviour controlled through the use of different solvents. ${ }^{17}$ Polyelectrolyte brushes have been used to mediate reversible adhesion through changing environmental $\mathrm{pH}^{\mathbf{1 8 , 1 9}}$ and ionic strength. ${ }^{20}$

Friction force microscopy (FFM, also known as lateral force microscopy) is an increasingly popular tool to analyse the mechanical and adhesive properties of a surface, in particular where a well-defined contact is desired..$^{21,22}$ The versatility of FFM is indicated by its application to different areas, such as the chemical, ${ }^{23,24}$ mechanical, ${ }^{24,25}$ and electrostatic ${ }^{26}$ characterisation of surfaces.

The combination of polyelectrolyte brushes and FFM offers a useful means of testing the material properties of surfaces. For example, a swollen brush should be expected to exhibit material properties different from a collapsed brush. The combination of JKR and DMT models should therefore allow a test of brush stiffness, with JKR behaviour indicating a more deformable contact and the DMT model indicating stiffer brushes. In this work, different FFM tips have been used: as-supplied silicon nitride tips, tips coated with gold, and gold-coated tips upon which a self-assembled monolayer (SAM) of mercaptoundecanoic acid (MUA) or dodecanethiol (DDT) was allowed to form. The DDT SAM represents an ideal hydrophobic surface, whilst gold represents a (charge) neutral surface. The MUA and silicon nitride tips are both hydrophilic, although the MUA is more hydrophilic than silicon nitride. It is shown that FFM can be used to determine material properties when measuring the frictional behaviour of a polymer brush, but also that it is important to account for the nature of the tip when interpreting the data.

\section{Experimental}

\section{Materials}

Silicon wafers (boron doped, $0-100 \Omega \mathrm{cm}$, and (100) orientation) were purchased from Prolog Semicor (Ukraine). Copper(I) chloride (99.999\%), copper(II) bromide (99.999\%), (11-(2-bromo-2-methyl)propionyloxy)undecyl trichlorosilane, 1-dodecanethiol (98\%), 11-mercaptoundecanoic acid (99\%), dry toluene (99.8\%), 2-(dimethyl amino)ethyl methacrylate $\left(\mathrm{C}_{8} \mathrm{H}_{15} \mathrm{NO}_{2}\right), \mathrm{HCl}(37 \%)$, and $\mathrm{NaOH}(>97 \%)$ were all purchased from Aldrich and used as received. HPLC grade acetone and methanol were purchased from Fisher Scientific. 2,2'-Dipyridyl (99\%) was purchased from Acros. Chromium chips (0.7-3.5 $\mathrm{mm}, 99.99 \%)$ were purchased from Agar and gold wire (99.99\% purity) was purchased from Testbourne.

\section{Brush synthesis}

Poly[2-(dimethyl amino)ethyl methacrylate] (PDMAEMA) was prepared on silicon substrates by atom transfer radical polymerization (ATRP). ${ }^{27}$ This is a "grafting from" method to synthesize the brush, and allows a controlled route to form densely grafted polymers on the surface. Here, the initiator was immobilized on the substrate, and then the brush was synthesized on the active surface.

To immobilize the initiator, first the silicon wafer was cleaned, homogenized, and rendered hydrophilic in oxygen plasma for $30 \mathrm{~min}$ at a pressure of $33 \mathrm{~Pa}$. In order to remove dust particles the wafers were cleaned by $\mathrm{N}_{2}$ gas. The clean surfaces were immersed in $20 \mathrm{~mL}$ of dry toluene solution containing 50 $\mu \mathrm{L}$ initiator for $6 \mathrm{~h}$ to immobilize the initiator. When coated, the substrates were removed from the solution and rinsed with toluene, and then dried under nitrogen gas.

For the PDMAEMA brush synthesis, 2,2'-dipyridyl (0.225 g), $\mathrm{CuCl}(0.0624 \mathrm{~g})$ and $\mathrm{CuBr}_{2}(0.0084 \mathrm{~g})$ were added together as catalysts. Degassed acetone $(15.9 \mathrm{~mL})$ and $1.5 \mathrm{~mL}$ deionized water were used to dissolve the catalysts for $1 \mathrm{~h} .10 .8 \mathrm{~mL} \mathrm{2-}$ (dimethyl amino)ethyl methacrylate (DMAEMA) was then injected into this solution under nitrogen. Finally, $20 \mathrm{~mL}$ of the solution was injected into a cell (sealed under nitrogen) which contained the initiator-coated silicon wafer. The reaction proceeded at $37^{\circ} \mathrm{C}$ for $16 \mathrm{~h}$. The samples were removed, rinsed with methanol and dried under nitrogen gas after reacting.

\section{Brush characterization}

Molecular weights and dispersities of free PDMAEMA were determined by gel permeation chromatography (GPC) relative to poly(ethylene oxide) standards at $70{ }^{\circ} \mathrm{C}$ using $\mathrm{N}, \mathrm{N}$-dimethylformamide and $0.1 \%$ ammonium acetate as the eluent at a flow rate of $1.00 \mathrm{~mL} \mathrm{~min}^{-1}$. To synthesize free PDMAEMA, $2 \mu \mathrm{L}$ of ethyl 2-bromoisobutyrate was dissolved in $48 \mu \mathrm{L}$ degassed acetone and then injected into the flask containing the DMAEMA. The synthesis continued as discussed above. After the reaction, the free PDMAEMA was characterized by GPC.

The molecular weight of PDMAEMA was determined to be 39 $\mathrm{kg} \mathrm{mol}{ }^{-1}$ by GPC, from which a grafting density of 0.84 chains per $\mathrm{nm}^{2}$ was calculated, assuming a density of $1 \mathrm{~g} \mathrm{~cm}^{-3}$. The dispersity was determined to be 1.37 . The average thickness of films of both dry PDMAEMA as well as those immersed in different $\mathrm{pH}$ solutions was determined by ellipsometry. The $\mathrm{pH}$ was routinely monitored with a $\mathrm{pH}$ meter. X-ray photoelectron spectroscopy was used to monitor each stage of the process to ensure that we had the expected chemistry on the surface, before and after deposition of the initiator layer, and after the polymerization reaction had completed.

\section{Friction force microscopy measurements}

A Digital Instruments Nanoscope IIIa Multimode atomic force microscope was used for friction force measurements operating in contact mode with a liquid cell/tip holder. FFM experiments were performed at a scan rate (constant tip velocity of $2 \mu \mathrm{m} \mathrm{s}^{-1}$ ) of $1 \mathrm{~Hz}$ with 256 points per $(1 \mu \mathrm{m})$ line. The frictional behaviour 
of the PDMAEMA brush was determined with non-conductive silicon nitride triangular probes (MLCT, Brucker) with a nominal spring constant of $0.065 \mathrm{~N} \mathrm{~m}^{-1}$ and a radius of $20 \mathrm{~nm}$. The spring constant of each cantilever was calibrated using a Digital Instruments PicoForce module and its associated software, based on the method of Hutter and Bechhoefer. ${ }^{28}$ The lateral force was calibrated using the wedge method, ${ }^{29-31}$ with the cantilever scanning across a calibration grating (TGF11, MikroMasch, Tallinn, Estonia). The frictional behaviour of each sample was measured in deionized water and solution with different $\mathrm{pH}(\mathrm{pH}=1$ to 12$)$ by the addition of $\mathrm{HCl}$ or $\mathrm{NaOH}$ as appropriate. All silicon nitride probes were cleaned with piranha solution, which is a mixture of $30 \%(15 \mathrm{~mL})$ hydrogen peroxide and $70 \%(35 \mathrm{~mL})$ sulfuric acid. Because piranha solution is a strong oxidizing agent, great care must be taken when handling it. They were finally rinsed with deionized water and were dried in an oven at $\sim 80^{\circ} \mathrm{C}$.

To determine the transition parameter, data were fitted to eqn (4) using $a_{0}, \alpha$ and $N_{\mathrm{PO}}$ as floating parameters. The contact area is directly proportional to the photodetector response, so the ratio of the photodetector response under applied load to that without load is equal to the square of eqn (4).

\section{Modification of the cantilevers}

The cantilevers were coated with a layer of gold by an Edwards Auto 306 bell jar vacuum coater system. To coat the tips with gold, a $2 \mathrm{~nm}$ thick layer of chromium was deposited at a rate of less than $0.05 \mathrm{~nm} \mathrm{~s}^{-1}$. These were then coated with $10 \mathrm{~nm}$ of gold at a rate of $0.03 \mathrm{~nm} \mathrm{~s}^{-1}$.

To modify the cantilevers with a SAM, the gold-coated tips were modified with DDT and MUA monolayers. To modify the tips with DDT, gold-coated tips were immersed in a mixture of $1 \mathrm{mM}$ 1-dodecanethiol and $20 \mathrm{~mL}$ of degassed ethanol and left for 12 hours. These were then rinsed in degassed ethanol.

Tip modification with an MUA monolayer was performed using the same procedure as for DDT, except that $5.5 \mathrm{mg}$ of 11mercaptoundecanoic acid was mixed with $20 \mathrm{~mL}$ of degassed ethanol.

\section{Results}

\section{Brush thickness}

To determine the thickness of the dry brush, a sample was scratched with a scalpel, and the height profile across the scratch was measured by scanning force microscopy (SFM) using the same non-conductive silicon nitride tips that were used for the FFM measurements. The thickness was determined to be $68 \mathrm{~nm}$, which was in agreement with separate spectroscopic ellipsometry measurements. Ellipsometry measurements were taken using wavelengths from 200 to $1000 \mathrm{~nm}$ and the data were fitted using the analysis software WVASE32 (JA Woollam). These experiments revealed a thickness of $66 \mathrm{~nm}$, and an oxide layer thickness of $2 \mathrm{~nm}$. Enough measurements were performed so that statistical errors in the SFM and ellipsometry measurements were less than $1 \mathrm{~nm}$.
The thickness of the brush was also measured in solutions of different $\mathrm{pH}$ by the addition of $\mathrm{HCl}$ or $\mathrm{NaOH}$ as appropriate using both SFM (in contact mode) and ellipsometry. The results are shown in Fig. 1. At low pH, the thickness of the brush layer was found to be greatest with values of $190 \mathrm{~nm}$ (SFM) and 176 $\mathrm{nm}$ (ellipsometry). As the $\mathrm{pH}$ was increased the thickness of the brush layer decreased, changing sharply between $\mathrm{pH} 3$ and 5, and then decreasing more slowly with increasing $\mathrm{pH}$. The brushes appear to be highly collapsed only at $\mathrm{pH} 12$. For most forms of brush profiles, ellipsometry is a more reliable measure of thickness than (contact mode) SFM because it is non-invasive. Nevertheless, there is reasonable agreement between the two techniques at all but the highest $\mathrm{pH}$ values.

The swelling of brushes of (similar) ATRP-grafted poly[2(diethyl amino)ethyl methacrylate] (PDEAEMA) has been studied by neutron reflectometry, ${ }^{32}$ and it was observed that these brushes, due to their rather dense grafting, experience counterion condensation, and do not swell particularly in pure water. This phenomenon is because the $\mathrm{p} K_{\mathrm{a}}$ of the brush layer is shifted to a smaller $\mathrm{pH}$ value than that for the free polymer in dilute solution. Although PDMAEMA is more hydrophilic than PDEAEMA, it should not be surprising that it also swells very little in water. Both PDEAEMA and PDMAEMA have very similar $\mathrm{p} K_{\mathrm{a}}$ values,${ }^{33}$ so it is reasonable to make the comparison between them.

\section{Adhesion}

Adhesion measurements were performed in solutions of different pH. 100 measurements were performed for each of the different tips at each pH. Example data for a DDT-coated tip for four different $\mathrm{pH}$ values are shown in Fig. 2. Approach curves can provide insight into the stiffness and modulus of the brush. $^{34}$ The retraction curves reveal raw adhesion data. Here the approach curves (Fig. 2a) show that there are no long-range repulsive or attractive interactions when the AFM tip approaches the polymer brushes, except for a large repulsion for the most extended brush at $\mathrm{pH} 1$. The stiffness of the brush layer, e.g. the sharp corner for $\mathrm{pH} \mathrm{4,} \mathrm{8,} \mathrm{and} \mathrm{12,} \mathrm{indicates} \mathrm{a} \mathrm{hard}$ surface while the slowly increasing repulsive force is a typical indicator for a swollen brush.

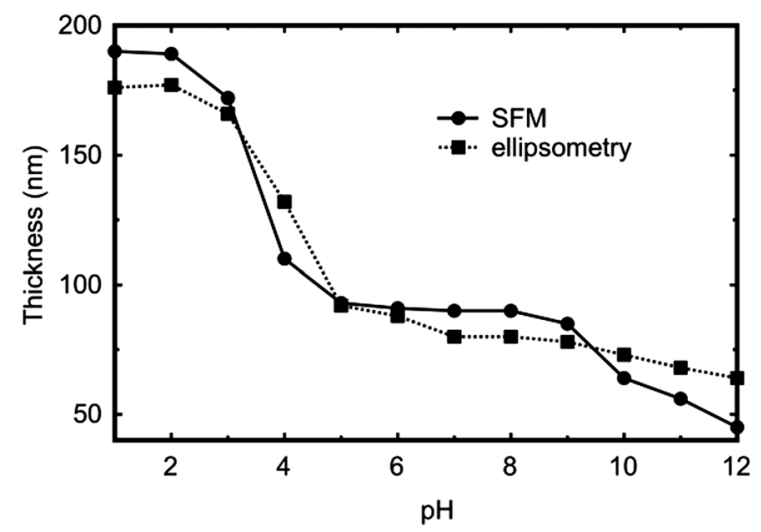

Fig. $1 \mathrm{pH}$ dependence of the thickness (as obtained using SFM and ellipsometry) of a $68 \mathrm{~nm}$ thick (when dry) PDMAEMA brush. 
(a)
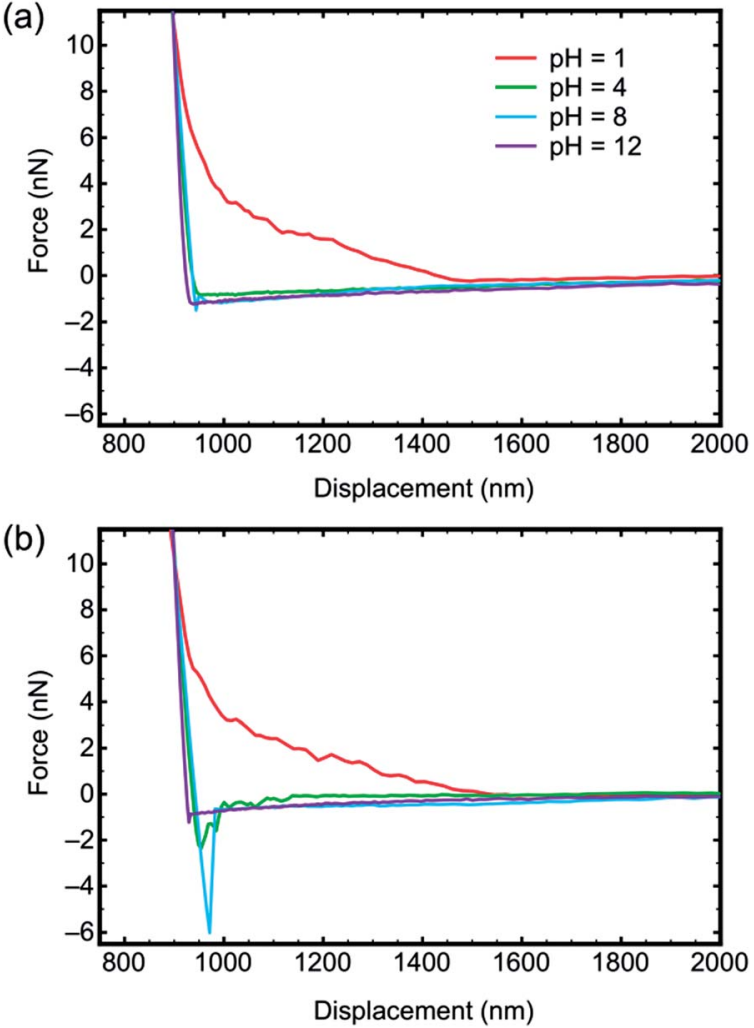

Fig. 2 Approach (a) and retraction (b) curves for a DDT-coated tip and the PDMAEMA brush for four different $\mathrm{pH}$ solutions.

The retraction curves (Fig. 2b) reveal the actual adhesion, which is defined as the minimum (most negative) force on these curves, and is very commonly referred to as the pull-off force. Here the data reveal very little interaction between the tip and the brush for $\mathrm{pH} 1$ and 12 . Indeed, these retraction curves are similar to the approach curves, further confirming the very limited interaction between these two components. Detailed histogram data for different $\mathrm{pH}$ for a gold-coated tip are presented in Fig. 3, and illustrate the distribution of adhesion events. The greatest mean adhesion was observed at $\mathrm{pH} 8$, when the distribution of adhesion is also the largest.

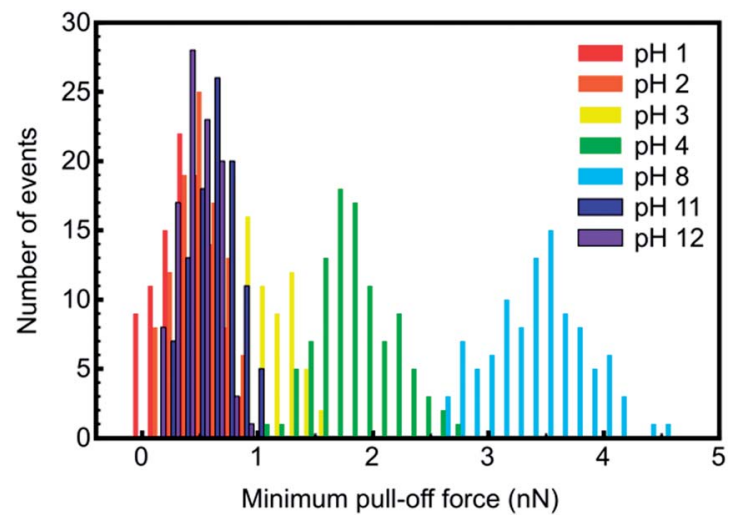

Fig. 3 Histograms of the minimum force required to separate a goldcoated tip from a PDMAEMA brush as a function of $\mathrm{pH}$.
Adhesion data for all tips and $\mathrm{pH}$ values are summarized in Fig. 4. For each tip coating, adhesion was always at its maximum value at $\mathrm{pH}$ 8. The greatest adhesion measured was for the MUA tip with the PDMAEMA brush over the entire range of $\mathrm{pH}$, although the difference was less pronounced at $\mathrm{pH} 1,2$, and 12 .

The large adhesion associated with MUA may be because it presents the most effective surface for hydrogen bonding with the PDMAEMA; gold and DDT should not permit hydrogen bonding at all, but silicon nitride should exhibit some interactions due to oxidation at its surface. Gold, silicon nitride, and DDT have similar values of adhesion over the different $\mathrm{pH}$ values. Silicon nitride has slightly larger values of the three at pH 3 and 4, indicating perhaps a role of charge in the adhesion, although it is surprising that silicon nitride does not have larger adhesion compared to gold and DDT due to the possible role of hydrogen bonding in the adhesion. At high pH (between 9 and 11), DDT has larger adhesion than gold and silicon nitride, and indeed has a value $(2.6 \mathrm{nN})$ approaching that of MUA $(3.8 \mathrm{nN})$ at $\mathrm{pH}$ 11, which might suggest that the alkyl chain has an important role in the interaction given that the uncharged carboxylic acid of the MUA and the methyl termination of DDT are quite dissimilar.

\section{Friction force microscopy}

Friction force microscopy was performed on PDMAEMA brushes as a function of $\mathrm{pH}$ (between 1 and 12 in steps of unity) and for different tip surface chemistries, specifically MUA, DDT, gold, and silicon nitride (i.e. the uncoated tip).

Fig. 5 contains representative friction-load plots obtained using a bare silicon nitride probe. It can be seen that a variety of types of behaviour was observed. At $\mathrm{pH}$ values of 1,2 , and 12, the friction-load relationship was linear, but for values between 3 and 11 it was non-linear. Here, the friction-load relationship was analysed using the general transition equation to determine whether the data could be best fitted with JKR or DMT mechanics. The values of transition parameter $\alpha$ are shown in Fig. 6. At pH 6 and 7 it was found that $\alpha=1$ for all surfaces, indicating that the behaviour is consistent with the JKR model.

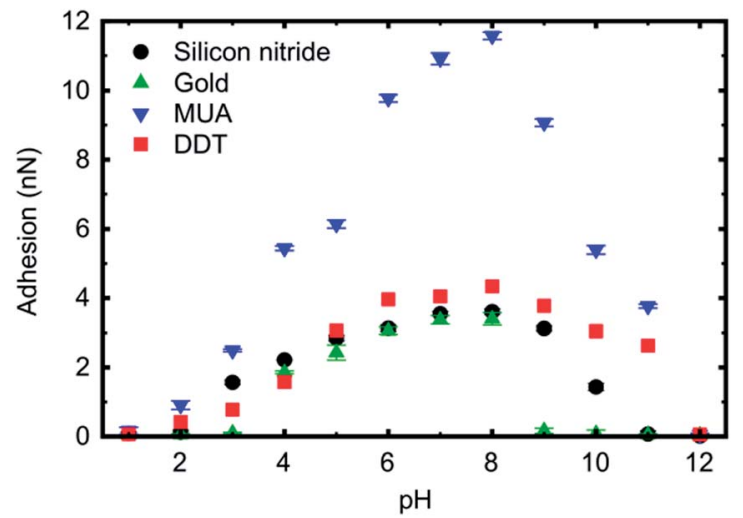

Fig. 4 Adhesion data for the different tips used in the present study. Error bars are included in the plot, but may be obscured by the symbol. 

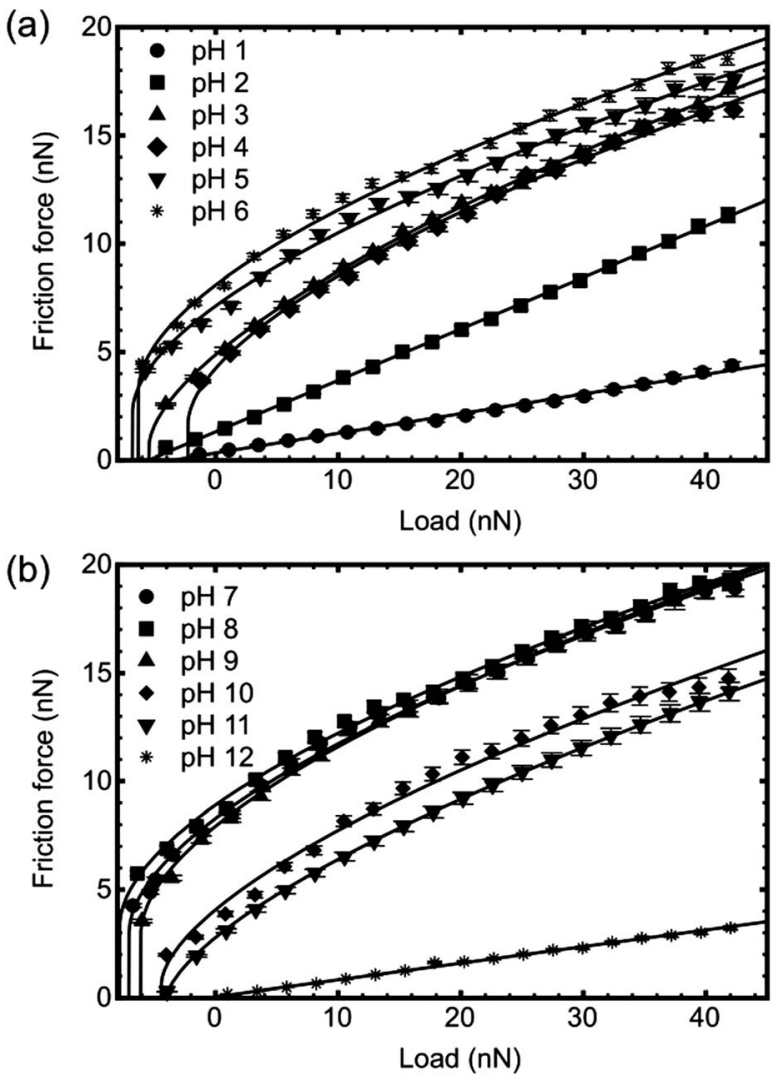

Fig. 5 Friction force as a function of load for a PDMAEMA brush in contact with an uncoated silicon nitride tip for different values of $\mathrm{pH}$. The solid lines are fits to the data, made by application of the general transition equation or Amontons' law, as described in the text. The error bars represent the average of three experiments on three different positions of each of three different surfaces; 27 measurements in total for each point.

At other $\mathrm{pH}$ values, intermediate behaviour was observed, or $\alpha=0$, indicating DMT behaviour.

The silicon nitride tip is weakly anionic, and so there is likely to be a region of $\mathrm{pH}$ where opposite charges are on the brush and on the tip. The large number of points where behaviour

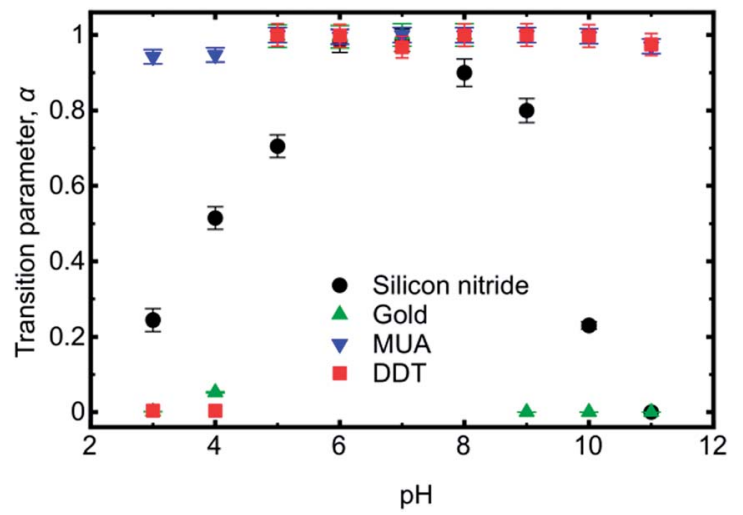

Fig. $6 \mathrm{pH}$ dependence of the transition parameter for the different tips in contact with a PDMAEMA brush. Although some data obscure each other, reasonable deduction can be used to see which is which; for example at $\mathrm{pH} 11, \alpha=0$ applies for the gold and silicon nitride tips. intermediate between JKR and DMT is observed may well be determined by the relative level of charging on the two surfaces. At these intermediate $\mathrm{pH}$ values the silicon nitride may still be negatively charged and the polymer may have a low density of positive charges; at lower $\mathrm{pH}$ the silicon nitride will become protonated and at higher $\mathrm{pH}$ the brush will have declining degrees of protonation. Certainly, similar arguments were used for the pH-dependence of silicon nitride AFM tips when in contact with a tungsten surface. ${ }^{35}$

For the gold-coated cantilever, the transition parameter was predominately close to zero, indicating DMT mechanics. Only over a narrow pH range, pH 5 to 8, was JKR behaviour observed. For gold, transition parameters intermediate between JKR and DMT were not observed, except for perhaps $\mathrm{pH} 4$, where $\alpha=$ 0.05 was obtained.

The DDT-coated cantilever exhibited JKR behaviour over most of the $\mathrm{pH}$ range, with only $\mathrm{pH} 3$ and 4 having $\alpha=0$, and DMT mechanics. As for the gold-coated cantilever, behaviour intermediate between JKR and DMT mechanics was not observed.

The most hydrophilic cantilever, i.e. the cantilever coated with MUA, exhibited JKR behaviour for all pH between 3 and 11, with only slight departures at $\mathrm{pH} 3,4$, and 11 ( $\alpha=0.94,0.95$, and 0.97 respectively). No DMT mechanics was observed at all with this cantilever. With the MUA-coated cantilever, it is expected that at least one component (brush or cantilever) was charged at all values of $\mathrm{pH}$ studied.

In the $\mathrm{pH}$ range between 3 and 11 only the MUA-coated tip has the same behaviour (JKR mechanics) for all solutions, with the other three exhibiting some form of $\mathrm{pH}$-dependent behaviour.

\section{Discussion}

\section{Tip-sample interactions}

The interaction between the PDMAEMA brush and the tip is complicated by the nature of the tip itself. Silicon nitride and MUA tips are negatively charged at high pH; gold, being a metal should not retain a charge, and DDT will be uncharged and hydrophobic.

The DDT tip exhibits JKR behaviour $(\alpha=1)$ over much of the $\mathrm{pH}$ range $(5 \leq \mathrm{pH} \leq 11)$ with DMT applicable for $\mathrm{pH} 3$ and 4 . At these $\mathrm{pH}$ values where DMT behaviour is observed, the PDMAEMA brush was extended (Fig. 1) and its state of solvation could perhaps explain the stiffness inherent in DMT behaviour. At larger values of $\mathrm{pH}(>5)$, the brush is more compressible, and here JKR behaviour is observed. It might be considered counterintuitive that a more extended brush is effectively stiffer than the one with a less extended conformation, but the pressure exerted by the counterions within the brush should convey stiffness. The adhesion data shown in Fig. 2a confirm that the interaction of the DDT-coated tip is one in which the brush may be treated as stiff at both $\mathrm{pH} 4$ and $\mathrm{pH} 8$.

For gold-coated tips, DMT behaviour was similarly observed for $\mathrm{pH} 3$ and 4 , and also for $9 \leq \mathrm{pH} \leq 11$. At low $\mathrm{pH}$, we can ascribe the same explanation for the contact mechanics as for the DDT-coated tip. At pH 10 and 11 the PDMAEMA brush is 
close to its dry thickness (Fig. 1), and may therefore be stiff at these $\mathrm{pH}$ values. It is harder to explain the result for $\mathrm{pH}$ 9, except to speculate that this might reflect the uncertainty in the measurements.

Given the gold and DDT results, one can suggest that the $\mathrm{p} K_{\mathrm{a}}$ of the brush is given by $4<\mathrm{p} K_{\mathrm{a}}<5$, which is somewhat lower than that in dilute solution ${ }^{33}(\sim 7.0)$, but is consistent with the behaviour of PDEAEMA brushes. ${ }^{32}$ There is a contradiction between the gold and DDT brushes for high $\mathrm{pH}$; if the brush is stiff due to it being compressed close to its dry thickness, then DMT behaviour should be expected for the DDT-coated tip at these $\mathrm{pH}$ values. The adhesion data (Fig. 4) show that gold has only a minimal interaction with the brush from $\mathrm{pH} 9$ to 11 , whereas the DDT has much stronger adhesion to the brush over this $\mathrm{pH}$ range. The origin of this adhesion is unclear, but it should be linked to the JKR contact mechanics of the tip and brush.

MUA-coated tips show JKR mechanics over the entire $\mathrm{pH}$ range, except for the extremes where a linear friction-load relationship was observed. The JKR mechanics indicates an intimate interaction with the brush. When the MUA and the brush are both charged, they adhere. However, if hydrogenbonding sites are available, then such an adhesive interaction can take place over a wider range of $\mathrm{pH}$, which would provide a plausible explanation for the wide range of $\mathrm{pH}$ over which JKR behaviour is observed. It is not clear whether hydrogen bonding, if responsible for the JKR mechanics, would be at different sites for high and low $\mathrm{pH}$, or at the same site for all $\mathrm{pH}$ values. If the same hydrogen bonding occurs over all $\mathrm{pH}$, it cannot be due to the carboxylic group on the acid, or the nitrogen on the base. The adhesion data (Fig. 4) certainly indicate a much stronger interaction between these two components than the brush with any other tips. Indeed MUA has a stronger adhesion to the brush between $\mathrm{pH} 4$ and 10 than any other sample.

If hydrogen bonding provides the explanation for the JKR mechanics over all $\mathrm{pH}$ values for the MUA-coated tip, then silicon nitride, which is not expected to be capable of interacting through hydrogen bonding should demonstrate DMT behaviour at both high and low $\mathrm{pH}$, which it does. Here, the silicon nitride tip can be expected to have a negative charge at high $\mathrm{pH}$, and so an adhesive interaction may result when both are charged. In acidic solution the brush is hydrated and DMT contact mechanics results, whereas in alkaline solution $(\mathrm{pH} 10$ and 11), the tip is solvated but the brush is collapsed, and is therefore stiffer.

The extremes of $\mathrm{pH}(1,2$, and 12) exhibit linear friction-load relationships, which might be expected for a weak interaction between the brush and the tip. This can certainly be expected for highly solvated brushes (i.e. $\mathrm{pH} 1$ and 2) but is perhaps surprising for $\mathrm{pH} 12$. The system in $\mathrm{pH} 2$ yielded the largest friction coefficient of the three that exhibited linear frictionload relationships, which indicates a slightly stronger interaction between the brush and the tip, and this is certainly to be expected when compared to the results for $\mathrm{pH} 1$. However, the system at $\mathrm{pH} 12$ exhibited the smallest friction coefficient for all of the tips, which implies it is energetically more beneficial for the tips to remain solvated than to be in contact with the polymer. The adhesion data (Fig. 4) certainly indicate a stronger interaction at $\mathrm{pH} 2$ than for $\mathrm{pH} 1$ and 12. The PDMAEMA is expected to be solvated at high $\mathrm{pH}$, in comparison with PDEAEMA, which is hydrophobic in such environments. ${ }^{36}$ Nevertheless, pH 12 is an extreme environment for the brush, and so may confer hydrophobicity to PDMAEMA. Certainly the thickness results (Fig. 1) indicate that the brush is hydrophobic at $\mathrm{pH} 12$.

In general, we cannot use the interaction between the brush and the SFM tip as a means to decipher material properties of the brush, such as whether or not it is swollen; we have seen that DMT behaviour can be observed for both collapsed and swollen brushes. Nevertheless, the gold and DDT results together do suggest that the brush starts to swell at $\mathrm{pH} \approx 4$. Recent experiments ${ }^{37}$ between SAM-coated AFM tips and a polyzwitterionic brush have shown that a linear friction-load relationship can exist in the case of interactions between the tip and the brush in both good and poor solvents. This suggests that the two different mechanisms giving rise to linear frictionload relationships represent reproducible phenomena in different systems.

The role of counterions in the brush stiffness is a consideration for asymmetries in the data (Fig. 6). At high $\mathrm{pH}$, the brushes are neutral and counterions play no role in the friction behaviour. At low $\mathrm{pH}$, the brushes are extended, but may nevertheless exhibit counterion condensation under compression. ${ }^{38,39}$ This alters the behaviour of the brushes, because the osmotic pressure of counterions acts to keep brushes elongated, which affects its stiffness and ability to resist deformation by the probe. Unfortunately, the level of counterion condensation taking place under these pressures is currently indeterminate, although future developments here would help us to understand the role of counterions on the molecular mechanisms of friction in much greater detail.

\section{Contact mechanics}

Single asperity mechanics has its roots in thermodynamics. The interaction is between two surfaces, each with their surface or interfacial energies, and the energy of interaction thus increases with the size of the contact. ${ }^{40}$ However, Amontons' behaviour is based on the notion of plastic deformation occurring between contacting surfaces that consist of multiple asperities. It has also been widely used, paradoxically, to model FFM data. One resolution to this apparent conflict is found in the long-established notion that friction can be treated as the sum of two components: adhesion (shearing) and ploughing. At the nanometre scale, Carpick has proposed that the friction may be treated as the sum of a pressure-dependent ploughing term and an area-dependent adhesion term, recently used to model a variety of data from monolayer systems: $:^{\mathbf{4 0 , 4 1}}$

$$
F=\mu\left(N+N_{\mathrm{PO}}\right)+\tau \pi\left(\frac{R\left(N+N_{\mathrm{PO}}\right)}{K}\right)^{2 / 3} .
$$

Here $\tau$ is a parameter known as the surface shear strength, and $K$ is the elastic modulus. The first, load-dependent term 
describes energy loss through either molecular deformations or through other means. Changes in the $\mathrm{pH}$ affect hydration and thus the local brush density, which is expected to change friction. Furthermore, the $\mathrm{pH}$ may also affect the tip, and so the interaction between the brush and the tip will be modified by environmental changes. In polar liquids for systems in which hydrogen bonding is expected to dominate, any adhesive contribution is negligible,$^{40-42}$ and hence the second term in eqn (5) may be neglected. Moreover, the pull-off force in the first term will be negligible, yielding a linear friction-load relationship that appears to be consistent with Amontons' law.

\section{Conclusions}

We have measured the frictional behaviour of PDMAEMA brushes in aqueous solution as a function of $\mathrm{pH}$ in response to perturbation by a model single asperity contact and have observed a range of behaviours including DMT, JKR contact mechanics, and a regime at the extremes of $\mathrm{pH}(1,2$, and 12) where Amontons' law is observed to apply. JKR behaviour is observed in all cases for the brushes in neutral or close to neutral solutions indicating that the polymer brush behaves as a soft elastic medium. Depending on the tip chemistry, at $\mathrm{pH}$ values either side of this region, the contact mechanics may be either DMT or JKR, indicating that the physical interaction between the brush and the tip is crucial in determining the frictional behaviour; DMT behaviour is notable for gold-coated and uncoated silicon nitride tips. Amontons' behaviour at $\mathrm{pH} 1$, 2 , and 12 suggests a very weak interaction between the tip and the brush and so a dissipative interaction is taking place. The nature of this dissipative interaction (ploughing) need not be the same. At $\mathrm{pH} 1$ and 2, the tip may well penetrate and deform the highly solvated brush, but at $\mathrm{pH} 12$ this is less likely because the brush is expected to have a relatively collapsed conformation. Nevertheless, at this $\mathrm{pH}$ some form of energy loss due to deformation of the polymer is likely.

\section{Acknowledgements}

The Engineering and Physical Sciences Research Council (Grant $\mathrm{EP} / 039999 / 1)$ is acknowledged for financial support.

\section{Notes and references}

1 G. Amontons, Hist. Acad. R. Sci., 1699, 206-227.

2 F. P. Bowden and D. Tabor, The Friction and Lubrication of Solids, Oxford, Oxford, 1954.

3 K. L. Johnson, K. Kendall and A. D. Roberts, Proc. R. Soc. London, Ser. A, 1971, 324, 301-313.

4 B. V. Derjaguin, V. M. Muller and Y. P. Toporov, J. Colloid Interface Sci., 1975, 53, 314-326.

5 D. Maugis, J. Colloid Interface Sci., 1992, 150, 243-269.

6 R. W. Carpick, D. F. Ogletree and M. Salmeron, J. Colloid Interface Sci., 1999, 211, 395-400.

7 H. Hertz, J. Reine Angew. Math., 1881, 92, 156-171.

8 D. Bureau and L. Léger, Langmuir, 2004, 20, 4523-4529.

9 T. Drobek and N. D. Spencer, Langmuir, 2008, 24, 1484-1488.
10 L. Léger, E. Raphaël and H. Hervet, Adv. Polym. Sci., 1999, 138, 185-225.

11 F. T. Limpoco, R. C. Advincula and S. S. Perry, Langmuir, 2007, 23, 12196-12201.

12 M. Kobayashi and A. Takahara, Chem. Rec., 2010, 10, 208216.

13 Y. Ohsedo, R. Takashina, J. P. Gong and Y. Osada, Langmuir, 2004, 20, 6549-6555.

14 J. B. Sokoloff, J. Chem. Phys., 2008, 129, 014901.

15 M. Ballauff and O. Borisov, Curr. Opin. Colloid Interface Sci., 2006, 11, 316-323.

16 M. Chen, W. H. Briscoe, S. P. Armes and J. Klein, Science, 2009, 323, 1698-1701.

17 Z. Zhang, A. J. Morse, S. P. Armes, A. L. Lewis, M. Geoghegan and G. J. Leggett, Langmuir, 2011, 27, 2514-2521.

18 R. La Spina, M. R. Tomlinson, L. Ruiz-Pérez, A. Chiche, S. Langridge and M. Geoghegan, Angew. Chem., Int. Ed., 2007, 46, 6460-6463.

19 G. Sudre, L. Olanier, Y. Tran, D. Hourdet and C. Creton, Soft Matter, 2012, 8, 8184-8193.

20 M. Kobayashi, M. Terada and A. Takahara, Soft Matter, 2011, 7, 5717-5722.

21 B. D. Beake and G. J. Leggett, Phys. Chem. Chem. Phys., 1999, 1, 3345-3350.

22 R. W. Carpick and M. Salmeron, Chem. Rev., 1997, 97, 11631194.

23 K. S. L. Chong, S. Sun and G. J. Leggett, Langmuir, 2005, 21, 3903-3909.

24 H. I. Kim and J. E. Houston, J. Am. Chem. Soc., 2000, 122, 12045-12046.

25 F. Dinelli, C. Buenviaje and R. M. Overney, J. Chem. Phys., 2000, 113, 2043-2048.

26 A. Marti, G. Hähner and N. D. Spencer, Langmuir, 1995, 11, 4632-4635.

27 P. D. Topham, J. R. Howse, C. J. Crook, A. J. Parnell, M. Geoghegan, R. A. L. Jones and A. J. Ryan, Polym. Int., 2006, 55, 808-815.

28 J. L. Hutter and J. Bechhoefer, Rev. Sci. Instrum., 1993, 64, 1868-1873.

29 D. F. Ogletree, R. W. Carpick and M. Salmeron, Rev. Sci. Instrum., 1996, 67, 3298-3306.

30 E. Tocha, J. Song, H. Schönherr and G. J. Vancso, Langmuir, 2007, 23, 7078-7082.

31 M. Varenberg, I. Etsion and G. Halperin, Rev. Sci. Instrum., 2003, 74, 3362-3367.

32 M. Geoghegan, L. Ruiz-Pérez, C. C. Dang, A. J. Parnell, S. J. Martin, J. R. Howse, R. A. L. Jones, R. Golestanian, P. D. Topham, C. J. Crook, A. J. Ryan, D. S. Sivia, J. R. P. Webster and A. Menelle, Soft Matter, 2006, 2, 10761080.

33 V. Bütün, S. P. Armes and N. C. Billingham, Polymer, 2001, 42, 5993-6008.

34 E. Kutnyanszky and G. J. Vancso, Eur. Polym. J., 2012, 48, 8-15.

35 M. S. Lim, S. S. Perry, H. C. Galloway and D. C. Koeck, J. Vac. Sci. Technol. B, 2002, 20, 575-579. 
36 D. Dupin, S. P. Armes, C. Connan, P. Reeve and S. M. Baxter, Langmuir, 2007, 23, 6903-6910.

37 Z. Zhang, A. J. Morse, S. P. Armes, A. L. Lewis, M. Geoghegan and G. J. Leggett, Langmuir, 2013, 29, 10684-10692.

38 F. Goujon, A. Ghoufi, P. Malfreyt and D. J. Tildesley, Soft Matter, 2012, 8, 4635-4644.

39 L. Spirin and T. Kreer, ACS Macro Lett., 2013, 2, 63-66.
40 K. Busuttil, N. Nikogeorgos, Z. Zhang, M. Geoghegan, C. A. Hunter and G. J. Leggett, Faraday Discuss., 2012, 156, 325-341.

41 N. Nikogeorgos, C. A. Hunter and G. J. Leggett, Langmuir, 2012, 28, 17709-17717.

42 K. Busuttil, M. Geoghegan, C. A. Hunter and G. J. Leggett, J. Am. Chem. Soc., 2011, 133, 8625-8632. 